\title{
Performance Evaluation of the VISTA 500
}

Dae-Hyun Ko, EunJung Cho, Woochang Lee, Sail Chun, and Won-Ki Min Department of Laboratory Medicine, Asan Medical Center, University of Ulsan College of Medicine, Seoul, Korea

\author{
Corresponding author: \\ Won-Ki Min \\ Department of Laboratory \\ Medicine, Asan Medical Center, \\ 88 Olympic-ro 43-gil, Songpa-gu, \\ Seoul 05505, Korea \\ Tel: $+82-2-3010-4503$ \\ Fax: +82-2-478-0884 \\ E-mail: wkmin@amc.seoul.kr
}

\begin{abstract}
In this study, we evaluated the performance of a recently developed immunoassay analyser, the VISTA 500 (Siemens, Germany). Precision, linearity, and comparison studies were performed according to the Clinical and Laboratory Standards Institute guidelines. The test items evaluated included IgG, IgA, IgM, C3, C4, ceruloplasmin, prealbumin, transferrin, haptoglobin, rheumatoid factor, anti-streptolysin O, and cystatin C. Commercial control materials (BioRad Laboratories, USA), commercial linearity validation materials (Maine Standards, USA), and patient samples were used for the evaluation. For the correlation study, analysis with a BN-II nephelometer (Siemens) was used as a comparative method. Total coefficients of variation of analytes were found to be between $1.9 \%$ and $5.5 \%$. Results of the linearity evaluation were also acceptable for the range tested. Correlations with comparative methods were acceptable. The VISTA 500 analyser showed satisfactory analytical performance with respect to precision, linearity, and comparison. We conclude that the VISTA 500 is likely a good candidate as an immunology analyser.
\end{abstract}

(J Lab Med Qual Assur 2016;38:52-57)

Key Words: VISTA 500, Performance, Evaluation studies as topic
면역 측정장비인 Vista 500 (Siemens, Erlangen, Germany)의 측정 성능을 평가해보았다. 평가한 검사항목 은 IgG, IgA, IgM, C3, C4, ceruloplasmin, prealbumin, transferrin, haptoglobin, rheumatoid factor $(\mathrm{RF})$, antistreptolysin $\mathrm{O}(\mathrm{ASO})$, 그리고 cystatin $\mathrm{C}$ 로 총 12 종목이다. 평가는 정밀도, 직선성 및 기존 장비와의 상관성에 대하여 이 루어졌다. 정밀도는 세 가지 농도의 정도관리물질(BioRad Immunology Control Lot No 52490; BioRad Laboratories, Hercules, CA, USA)를 이용하여 평가하였다. Clinical and Laboratory Standards Institute (CLSI) EP05-A3에 따라 각각의 정도관리물질을 20 일 동안 매일 2 run씩, run당 2회씩 반복측정하여 평가하였다[1]. 직선성은 CLSI EP06-A에 따라 평가하였다[2]. 검사종목 중 $\operatorname{IgG}$, transferrin은 5가지 농도를 가진 Validate SP1 linearity test kit (Lot No 61AB07114; Maine Standards, Cumberland Foreside, ME, USA)를 이 용하여 평가하였고, C3, ceruloplasmin, prealbumin은 높 은 농도와 낮은 농도의 정도관리물질(BioRad Immunology Control Lot No 52490)을 이용하였고, 그 외의 종목들에 대
해서는 환자 검체 혼합물을 이용하여 평가하였다. 높은 농도 와 낮은 농도를 가진 검체 또는 정도관리물질을 혼합하여 5 가지 농도를 가진 혼합물을 만들어 이들을 각각 4회씩 반복 측정하여 그 결과를 이용하여 직선성을 평가하였다. 상관성 은 CLSI EP09-A2에 따라 기존 검사실에서 사용 중인 BN-II Nephelometer (Siemens)와 비교하였다[3]. 각 종목별로 다 양한 농도를 가진 40 개 이상의 환자 검체를 이용하여 두 장비 에서 각각 2 회씩 반복 측정하여 그 결과를 비교하였다. 각 종 목별 정밀도 평가결과를 Table 1에 나타내었다. Fig. 1은 직선 성 평가결과를 보여주는 그래프이고, Fig. 2는 상관성 평가결 과를 보여주고 있다. 각 항목별 정밀도 평가결과, total CV는 $1.9 \%$ 에서 $5.4 \%$ 수준으로 모두 만족할 만한 수준이었고, 직선 성도 평가한 범위 내에서 기울기 0.973-1.038, 절편 -1.6-0.0 으로 우수했다. 환자 검체를 이용한 기존 장비와의 비교결과도 R값이 0.9656-0.9985로 전반적으로 우수한 것으로 나타났다. 이상의 결과로 볼 때 VISTA 500은 임상검사실에서 면역 측정 장비로서 유용하게 사용할 수 있을 것으로 생각된다. 
Dae-Hyun Ko et al • Performance Evaluation of the VISTA 500

Table 1. Precision profile of the VISTA 500

\begin{tabular}{|c|c|c|c|c|c|c|}
\hline \multirow{2}{*}{ Analyte } & \multirow{2}{*}{ QC level } & \multirow{2}{*}{ Mean $(\mathrm{mg} / \mathrm{dL})$} & \multicolumn{4}{|c|}{ CV (\%) } \\
\hline & & & Within-run & Between-run & Between-day & Total \\
\hline \multirow[t]{3}{*}{ IgG } & Low & 774.5 & 2.2 & 0.9 & 1.0 & 2.5 \\
\hline & Middle & $1,355.0$ & 2.8 & 1.8 & 0.9 & 3.4 \\
\hline & High & $1,861.0$ & 2.7 & 1.4 & 1.5 & 3.4 \\
\hline \multirow[t]{3}{*}{$\operatorname{Ig} \mathrm{A}$} & Low & 124.8 & 2.2 & 1.0 & 0.7 & 2.6 \\
\hline & Middle & 259.5 & 1.8 & 1.5 & 1.3 & 2.7 \\
\hline & High & 392.5 & 2.5 & 1.2 & 1.4 & 3.1 \\
\hline \multirow[t]{3}{*}{$\operatorname{IgM}$} & Low & 61.3 & 3.3 & 0.0 & 1.9 & 3.8 \\
\hline & Middle & 140.0 & 2.7 & 1.5 & 2.3 & 3.9 \\
\hline & High & 215.1 & 3.3 & 1.7 & 1.7 & 4.1 \\
\hline \multirow[t]{3}{*}{$\mathrm{C} 3$} & Low & 85.9 & 1.9 & 0.0 & 0.9 & 2.1 \\
\hline & Middle & 170.2 & 2.1 & 0.9 & 0.8 & 2.4 \\
\hline & High & 248.4 & 2.7 & 1.7 & 0.0 & 3.2 \\
\hline \multirow[t]{3}{*}{$\mathrm{C} 4$} & Low & 16.3 & 1.7 & 0.8 & 0.0 & 1.9 \\
\hline & Middle & 33.2 & 1.8 & 1.4 & 0.7 & 2.4 \\
\hline & High & 49.5 & 2.8 & 0.9 & 0.7 & 3.0 \\
\hline \multirow[t]{3}{*}{ Anti-streptolysin $\mathrm{O}$} & Low & 87.8 & 5.4 & 0.0 & 0.5 & 5.4 \\
\hline & Middle & 145.1 & 4.3 & 1.1 & 1.9 & 4.8 \\
\hline & High & 193.5 & 3.5 & 0.4 & 2.4 & 4.3 \\
\hline \multirow[t]{3}{*}{ Ceruloplasmin } & Low & 19.4 & 2.0 & 0.8 & 0.0 & 2.2 \\
\hline & Middle & 35.7 & 2.6 & 0.9 & 0.0 & 2.7 \\
\hline & High & 51.2 & 1.8 & 1.1 & 0.0 & 2.1 \\
\hline \multirow[t]{3}{*}{ Cystatin C } & Low & 0.40 & 3.0 & 0.7 & 1.8 & 3.6 \\
\hline & Middle & 0.50 & 2.1 & 1.4 & 1.3 & 2.8 \\
\hline & High & 0.57 & 2.9 & 1.4 & 0.0 & 3.2 \\
\hline \multirow[t]{3}{*}{ Haptoglobin } & Low & 68.0 & 2.5 & 1.8 & 0.0 & 3.0 \\
\hline & Middle & 148.0 & 2.7 & 1.1 & 0.7 & 3.1 \\
\hline & High & 228.8 & 2.9 & 1.6 & 0.0 & 3.3 \\
\hline \multirow[t]{3}{*}{ Prealbumin } & Low & 14.6 & 3.0 & 1.2 & 0.0 & 3.2 \\
\hline & Middle & 25.4 & 3.4 & 0.0 & 1.9 & 3.8 \\
\hline & High & 36.2 & 3.4 & 0.8 & 0.9 & 3.6 \\
\hline \multirow[t]{3}{*}{ Rheumatoid factor } & Low & 14.0 & 4.2 & 0.0 & 1.5 & 4.4 \\
\hline & Middle & 20.2 & 2.6 & 1.5 & 0.0 & 3.0 \\
\hline & High & 25.2 & 2.5 & 1.1 & 0.0 & 2.8 \\
\hline \multirow[t]{3}{*}{ Transferrin } & Low & 155.4 & 2.6 & 0.0 & 0.8 & 2.8 \\
\hline & Middle & 258.0 & 3.4 & 1.6 & 0.0 & 3.8 \\
\hline & High & 350.0 & 3.1 & 0.0 & 0.0 & 3.1 \\
\hline
\end{tabular}


A
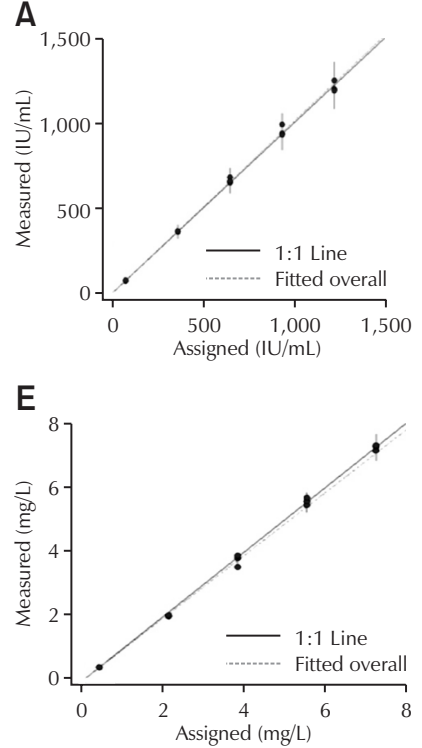

I

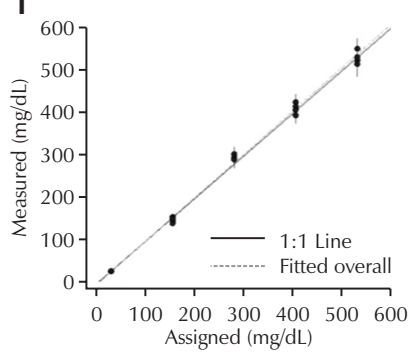

B

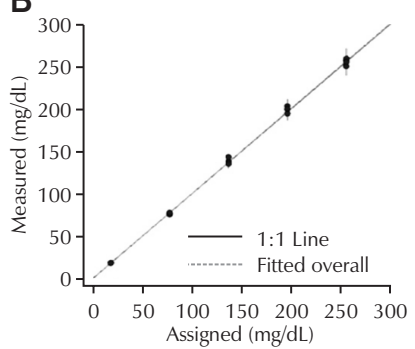

$\mathbf{F}$
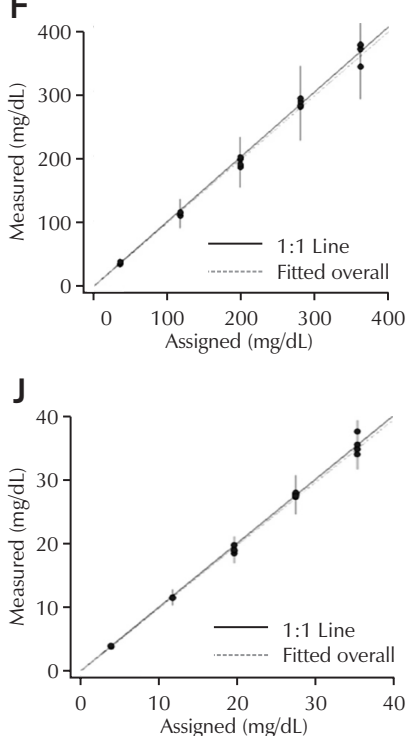

C

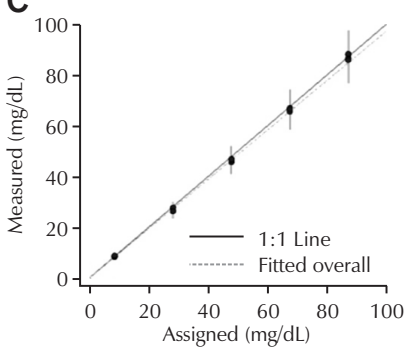

G

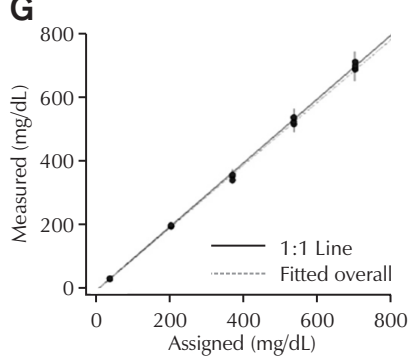

$\mathrm{K}$

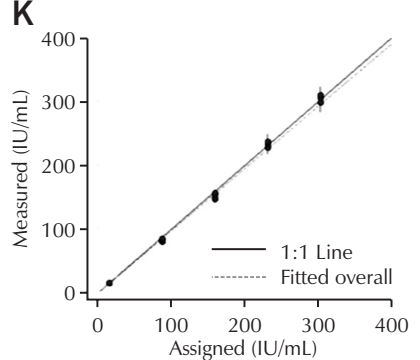

D

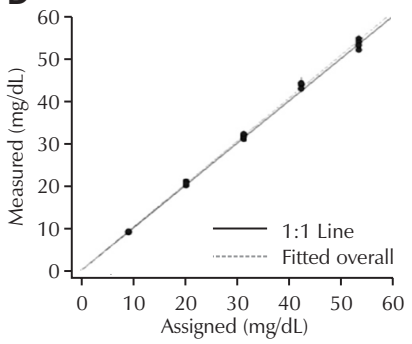

$\mathrm{H}$

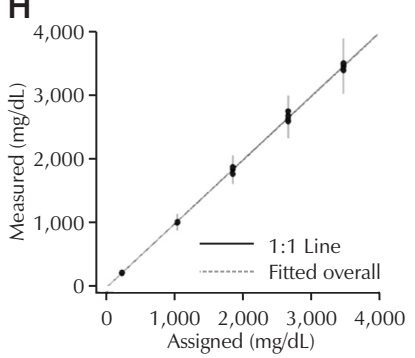

L

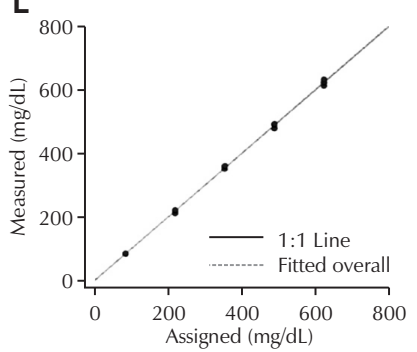

Fig. 1. Linearity studies using the VISTA 500. (A) Anti-streptolysin O. (B) C3. (C) C4. (D) Ceruloplasmin. (E) Cystatin C. (F) Haptoglobin. (G) Ig A. (H) Ig G. (I) Ig M. (J) Prealbumin. (K) Rheumatoid factor. (L) Transferrin. 
A

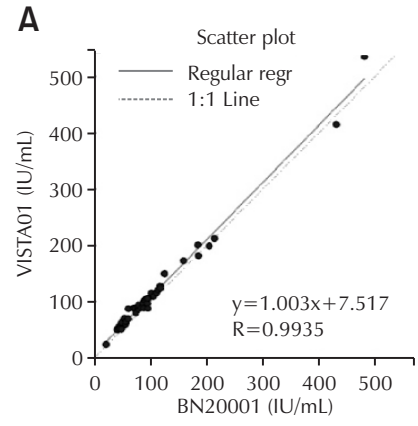

C

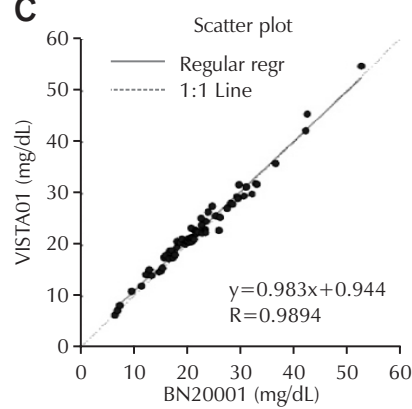

$E$

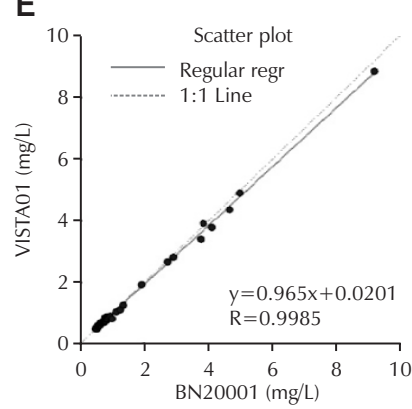

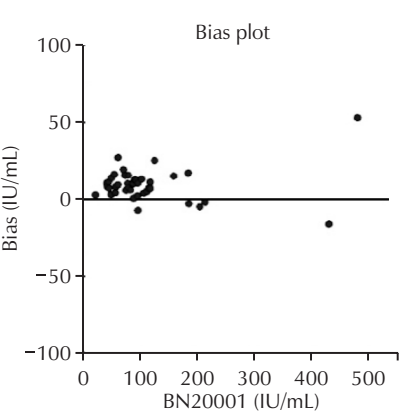
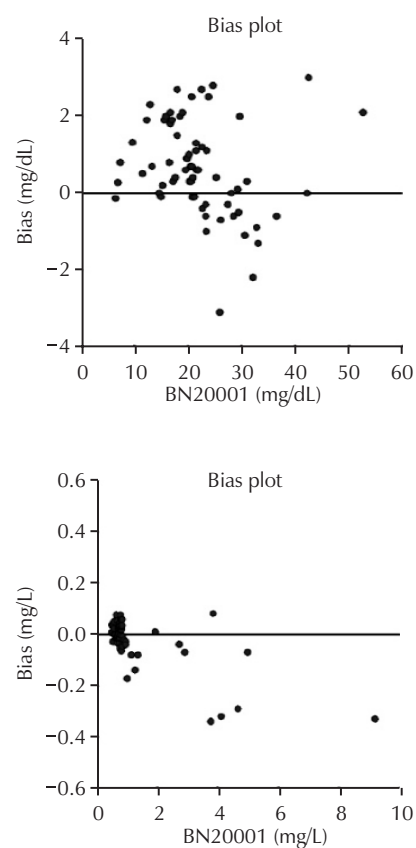

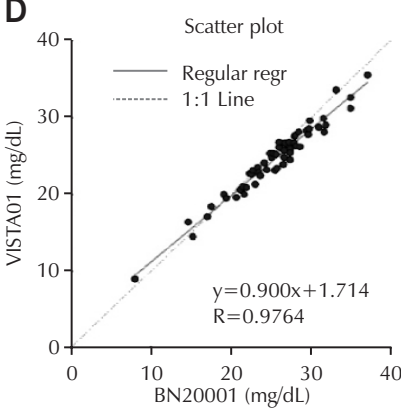

$\mathrm{F}$

B
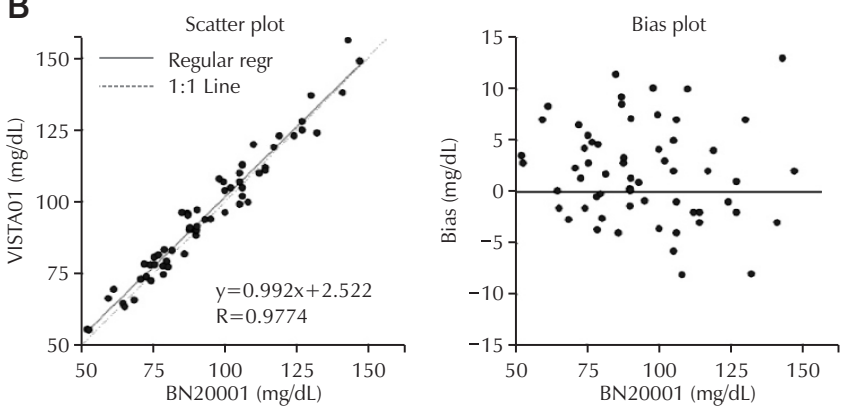

D

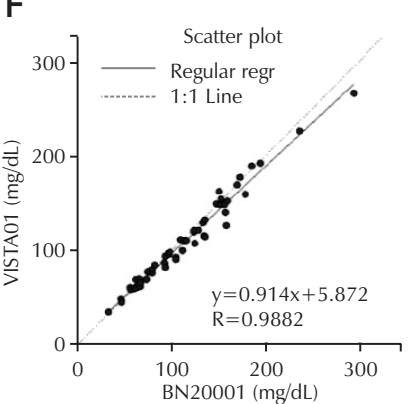

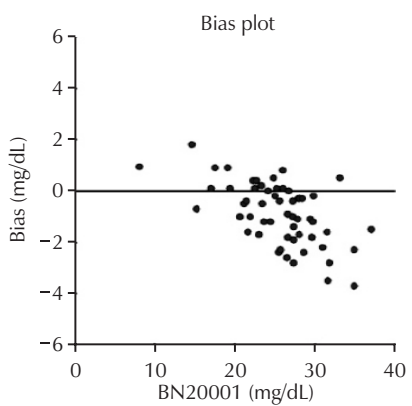

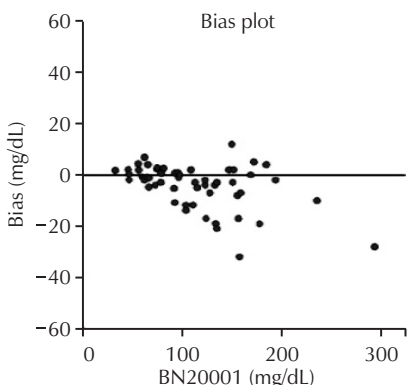

Fig. 2. Comparison of the VISTA 500 and BN-II nephelometer. For each analyte, a scatter plot (A) and bias plot (B) are shown. In scatter plots, solid lines represent regression lines and dotted lines represent identity lines. ASO, anti-streptolysin O; RF, rheumatoid factor. (A) Anti-streptolysin O. (B) C3. (C) C4. (D) Ceruloplasmin. (E) Cystatin C. (F) Haptoglobin. (G) Ig A. (H) Ig G. (I) Ig M. (J) Prealbumin. (K) Rheumatoid factor. (L) Transferrin. 
G

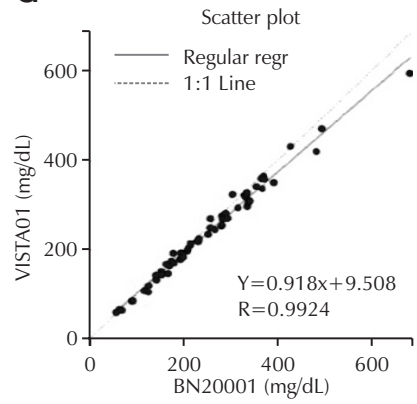

I
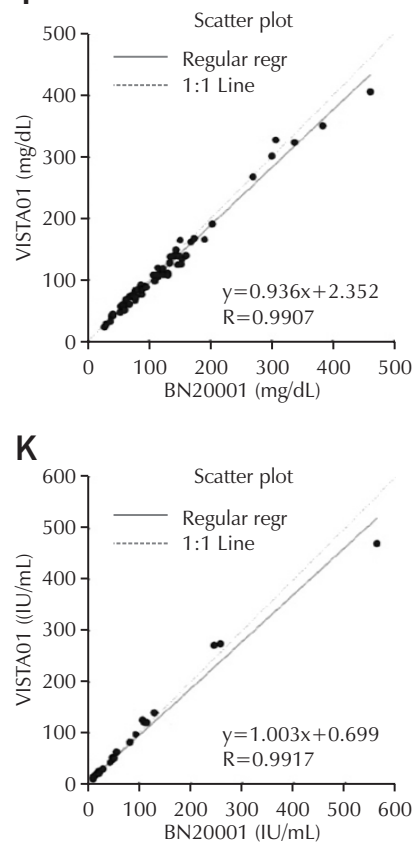
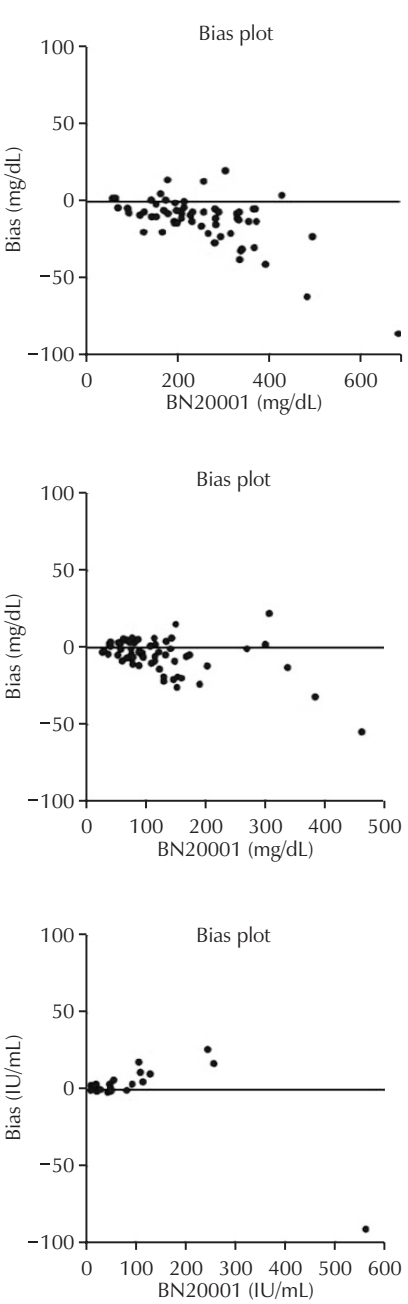

$\mathrm{H}$

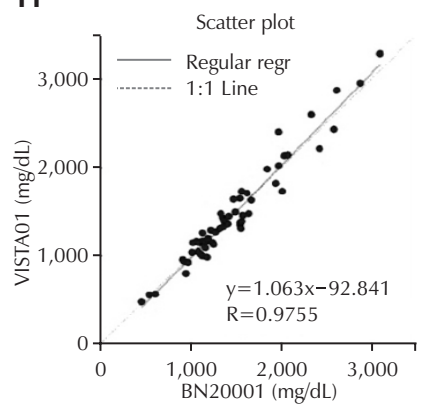

J
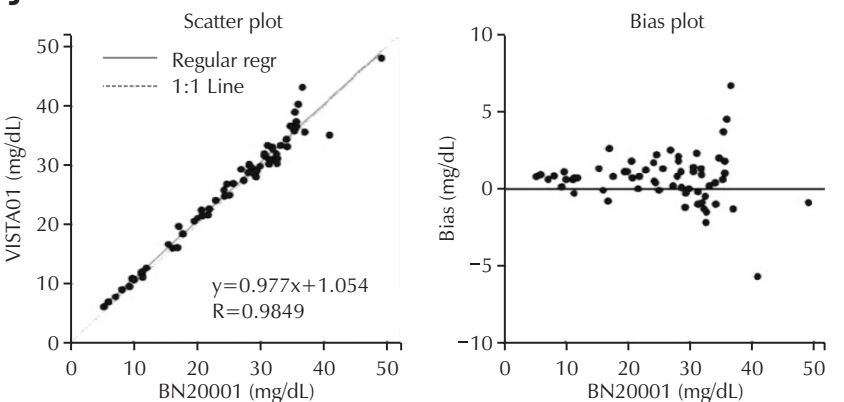

$\mathrm{L}$
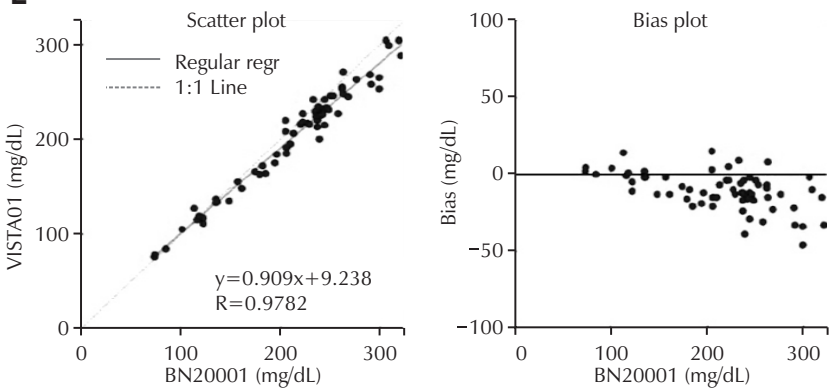

Fig. 2. Continued.

\section{REFERENCES}

1. Clinical and Laboratory Standards Institute. Evaluation of precision performance of quantitative measurement methods; approved guideline. 3rd ed. Wayne (PA): Clinical and Laboratory Standards Institute, 2014.

2. Clinical and Laboratory Standards Institute. Evaluation of the linearity of quantitative measurement procedures: a statistical approach: approved guideline. Wayne (PA): Clinical and Laboratory Standards Institute, 2003.

3. Clinical and Laboratory Standards Institute. Measurement procedure comparison and bias estimation using patient samples: approved guideline. 3rd ed. Wayne (PA): Clinical and Laboratory Standards Institute, 2013. 
VISTA 500 장비의 성능 평가 고대현 • 조은정 - 이우창 • 전사일 • 민원기

울산대학교 의과대학 서울아산병원 진단검사의학과

새로운 immunoassay analyzer인 VISTA 500 (Siemens, Germany)의 성능을 평가해 보았다. 정밀도, 직선성, 그리고 상관성을 Clinical and Laboratory Standards Institute 기준에 따라 평가 하였다. 평가한 검사종목은 $\mathrm{lgG}, \operatorname{lgA}, \operatorname{lgM}, \mathrm{C} 3, \mathrm{C} 4$, ceruloplasmin, preblaumin, transferrin, haptoglobin, rheumatoid factor, anti-streptolysin O, 그리고 cystatin C였다. BioRad Laboratories (USA), 상용화된 Maine Standards (USA)과 환자 검체가 평가에 사용되었다. 상관성 평가에서는 BN-II Nephelometer (Siemens)를 비교 장비로 사용하였다. 평가한 종목의 총 CV는 $1.9 \%-5.5 \%$ 로 나타났고, 직선성 결과도 평가한 범위 내에서 만족할 만한 수준이었다. 비교 장비와의

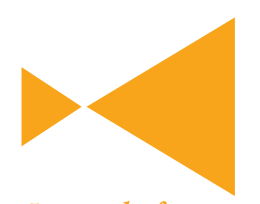

Journal of

LABORATORY MEDICINE and

QUALITY ASSURANCE 상관성도 우수한 성적을 보여주었다. VISTA 500은 정밀도, 직선성, 상관성 측면에서 우수한 분석 성 능을 보여주어 임상검사실에서 유용하게 사용할 수 있을 것으로 생각된다.

(J Lab Med Qual Assur 2016;38:52-57)

교신저자: 민원기

우05505 서울시 송파구 올림픽로 43길 88, 울산대학교 의과대학 서울아산병원 진단검사의학과

Tel: 02)3010-4503, Fax: 02)478-0884, E-mail: wkmin@amc.seoul.kr 